\title{
Tribological Properties of DLC and Sintered-Graphite-Carbon Sliding Pair ${ }^{*}$
}

\author{
Naofumi HIRAOKA ${ }^{* *}$, Nobuaki SAITO ${ }^{* *}$ and Yoshifumi ARIGA ${ }^{* * *}$ \\ ${ }^{* *}$ Institute of Technologists \\ 333 Maeya, Gyoda, Saitama 361-0038, Japan \\ E-mail: hiraoka@iot.ac.jp

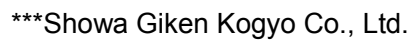 \\ 1247 Kobarishinjuku, Ina-cho, Kitaadachi-gun, Saitama 362-0808, Japan \\ E-mail: yariga@sgk-p.co.jp
}

\begin{abstract}
Friction and wear tests were conducted for two kinds of material combinations: a DLC-coated and sintered-graphite-carbon material combination, and a non-coated and sintered-graphite-carbon material combination, for comparison. DLC showed a higher friction coefficient than non-DLC materials. Carbon transfer films were only observed at certain places on the DLC surfaces, while most non-DLC surfaces were coated with those films. However, for DLC specimens, the high friction was found to be attributable to the transfer-film area but not non-transfer-film area (i.e. DLC itself). The sintered-graphite-carbon specimens having slid on DLC had little oriented surface layers due to anti-adhesion and amorphous properties of DLC while those having slid on non-DLC were strongly oriented to the sliding direction. Thus, the surface orientation was necessary for sintered carbon graphite to indicate low friction when sliding on its transfer film, and the high friction of DLC was due to the sliding between the little oriented sintered graphite carbon and the transfer film.
\end{abstract}

Key words: DLC, Sintered Carbon Graphite, Transfer Film, Mechanical Seal, Friction, Orientation

\section{Introduction}

Mechanical seals are excellent sealing devices especially for liquid sealing. These seals are sometimes used in the gas (e.g. air, steam) sealing often found in chemical plants. Under the dry conditions in such cases, the friction, wear, and surface damage of sealing surfaces remain of great concern. Therefore, proper combinations of sealing materials must be further investigated as possible alternatives to conventional combinations of ceramics or hard alloys and sintered graphite carbon.

DLC (Diamond Like Carbon) offers great potential as a tribological material. Moreover, its tribological performance has been greatly improved and DLC is already used in wide industrial applications (see Refs. (1) and (2)). We focused on DLC as a material for the sealing part of a mechanical seal as applied in particular to gas sealing and began investigating its friction and wear properties.

In this study, we investigated the tribological properties of DLC and a sintered-graphite-carbon combination in air to determine the performance of DLC as a direct alternative to conventional ceramics or hard alloys. Aside from potential application as a mechanical seal material, this combination posed two major issues of concern in terms of tribology: exfoliation of DLC and carbon-carbon sliding.

Although DLC is now being applied to such high contact pressure applications as the 
cam systems for automotive engines ${ }^{(3)}$ where strong anti-exfoliation of DLC is required, exfoliation remains a significant point requiring improvement for $\operatorname{DLC}^{(4)}$ since many applications still require more anti-exfoliation of DLC. Many techniques for anti-exfoliation of DLC are under development (e.g. using proper materials for an interface layer between DLC and the substrate $\left.{ }^{(1),(2)}\right)$. In application to mechanical seals, however, since the contact pressure is relatively low due to the plane contact of sealing surfaces and soft contact material of mating surfaces such as sintered graphite carbon, exfoliation is not expected to become a significant problem.

Sliding pairs of compatible materials generally exhibit unfavorable tribological performance with certain exceptions. The combination of DLC and sintered graphite carbon used in this study is also considered a compatible material combination: a carbon-carbon combination, though one side has an approximate diamond structure and the other side is almost totally graphite. Although there have been previous reports on the tribological properties of DLC and DLC ${ }^{(5)-(7)}$ or graphite and graphite sliding pairs ${ }^{(8)}$, our review found no such reports on DLC and graphite. In the case of this study, we expected either of the sliding between the sintered graphite carbon and strongly adhering carbon transfer film on DLC due to material compatibility or the sliding at the interface between the sintered graphite carbon and DLC due to anti-adhesion of DLC. In either case, relatively good performance could be expected.

\section{Experiment}

Table 1 lists the test materials. The sintered-graphite-carbon material of the lower specimen is hereafter designated "sintered carbon." Most of the volume of this specimen was graphite, and included an unknown percentage of non-graphitized carbon and impregnant. All surfaces of the upper specimens without DLC coating were lapped to a roughness of about Ra $0.2 \mu \mathrm{m}$. A coating of DLC about $1-\mu \mathrm{m}$ thick was applied to the materials, which were finished in the same manner as the specimens without DLC coating, by ionized vapor deposition using $\mathrm{C}_{6} \mathrm{H}_{6}$ gas. Hardness of the DLC coating was $\mathrm{Hv}$ $1800 \sim 2500$ for $\mathrm{S} 45 \mathrm{C}$ or SKD11 substrates and $\mathrm{Hv} 1000 \sim 1500$ for $\mathrm{Al}_{2} \mathrm{O}_{3}$ substrates. Hardness of DLC was different between conductive and nonconductive substrates. A silicon base interface layer of unknown detailed composition was applied between the substrate materials and DLC.

Table 1 Test materials

\begin{tabular}{|c|c|c|}
\hline No. & Upper specimen & Lower specimen \\
\hline 1 & $\mathrm{DLC}$ on $\mathrm{Al}_{2} \mathrm{O}_{3}$ & $\begin{array}{l}\text { Sintered-graphite-carbon impregnated with phenolic } \\
\text { resin }\end{array}$ \\
\hline 2 & DLC on JIS S45C & $\uparrow \uparrow$ \\
\hline 3 & $\begin{array}{l}\text { DLC on } \\
\text { heat treated JIS SKD11 }\end{array}$ & $\uparrow$ \\
\hline 4 & $\mathrm{DLC}$ on $\mathrm{Al}_{2} \mathrm{O}_{3}$ & Sintered-graphite-carbon impregnated with Antimony \\
\hline 5 & $\mathrm{Al}_{2} \mathrm{O}_{3}(\mathrm{Hv} 1000 \sim 1300)$ & Sintered-graphite-carbon impregnated with phenolic resin \\
\hline 6 & JIS S45C (Hv 330 360) & $\uparrow$ \\
\hline 7 & $\begin{array}{l}\text { Heat treated JIS SKD11 } \\
(\mathrm{Hv} 700 \sim 750)\end{array}$ & $\uparrow$ \\
\hline
\end{tabular}

A ring-on-ring friction and wear tester was used. The upper specimens (fixed specimens) had 18-mm inner and 26-mm outer diameters; the lower specimen (rotating specimens) had $18-\mathrm{mm}$ inner and $25.5-\mathrm{mm}$ outer diameters. The upper specimens were 
rinsed in ethanol and ultrasonically cleaned in acetone prior to the experiment and the lower specimens were used as received. The load was $204 \mathrm{~N}$ (about $0.8 \mathrm{MPa}$ in apparent contact pressure) and sliding velocity $0.75 \mathrm{~m} / \mathrm{s}$, typical application conditions for mechanical seals of similar size. All tests were conducted in laboratory air under 30 to $50 \% \mathrm{RH}$ and temperature of 20 to $24^{\circ} \mathrm{C}$. Tests for No.1, No.2, No.3 material combinations were conducted at 5, 2, 2 times, respectively. However, as the results were very similar, only a typical result for each combination is shown. Tests for No.4 to No.7 material combinations were conducted only once for each combination.

\section{Experimental Results}

Figure 1 shows the time evolution of friction coefficients. While the non-DLC specimens showed a relatively low friction coefficient of about 0.2 for five hours (Fig. 1(b)), the friction coefficient of DLC specimens fluctuated and increased, leading to the discontinuation of testing for a short time (Fig. 1(a)). The DLC specimens generated high frictional noise when the friction coefficient was high.

DLC was not exfoliated in tests No.1 to No.4. However, in another test of the DLC specimen that continued for five hours and of which result is not shown, the friction coefficient suddenly decreased to about 0.2 after a high duration of friction lasting a few tens of minutes. DLC was found exfoliated after the test, but this evidently occurred at the sudden decrease in friction.

Figure 2 shows the specific wear rates of the sintered-carbon specimens. Although the trends in friction coefficient were much different between the DLC and non-DLC specimens, the specific wear rates were not much more different than expected from the results of the friction coefficient, and all rates were between $(2 \sim 12) \times 10^{-6} \mathrm{~mm}^{3} /(\mathrm{Nm})$.
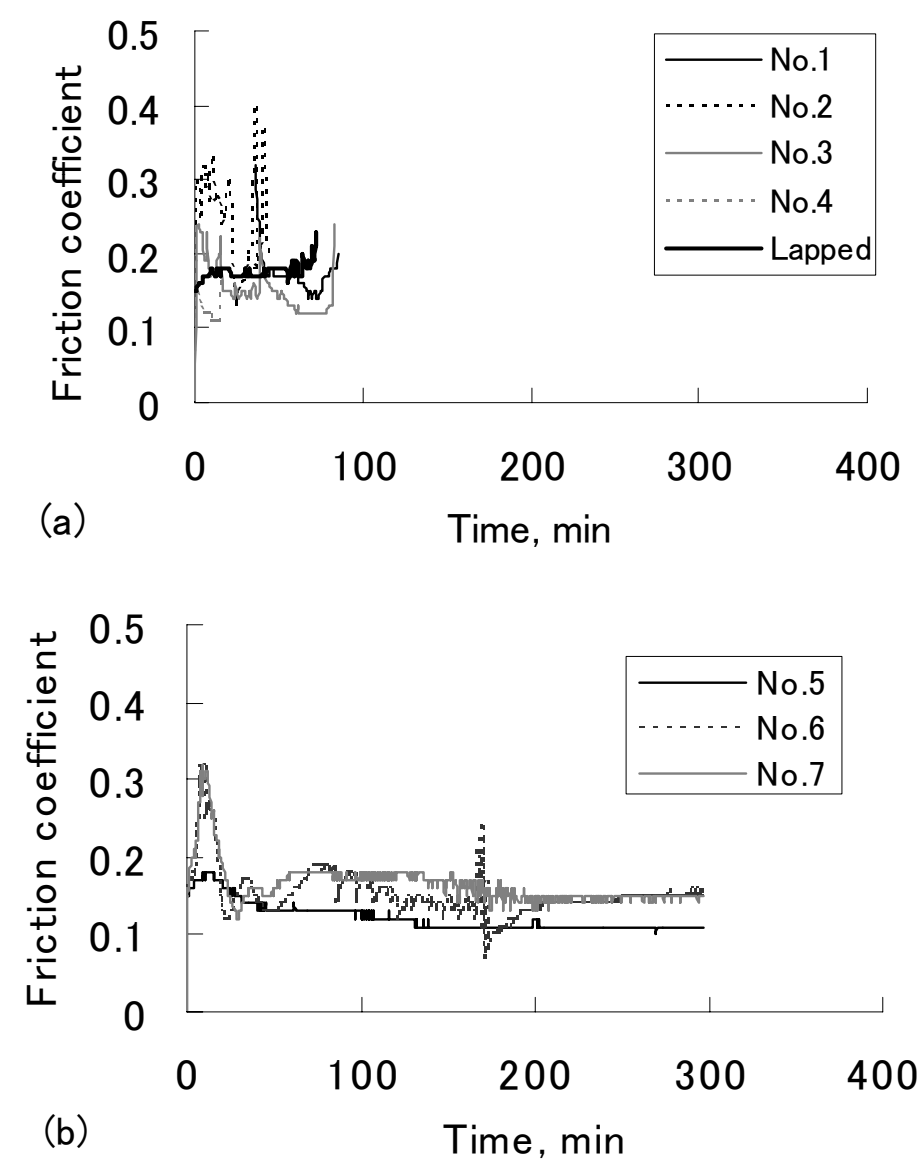

Fig. 1 Time evolution of friction coefficients 


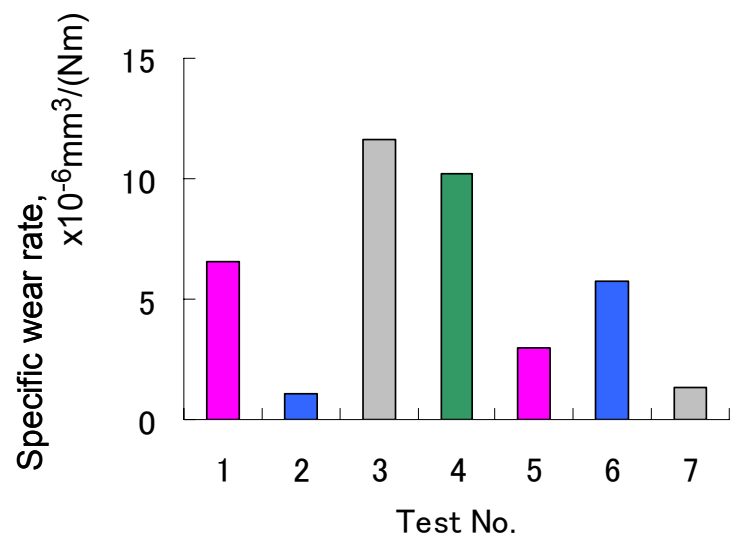

Fig. 2 Specific wear rates of sintered-carbon specimens

Figure 3 shows photos of the DLC and non-DLC specimen surfaces after the tests. The vertical direction slanting slightly toward the right in the photos approximately corresponds to the sliding direction. While the non-DLC specimen surface (Fig. 3(b)) appeared covered by thick, uniform transfer film from the mated sintered-carbon specimen, only relatively thin-looking transfer films (interference fringes, which were not clear in the photo, were observed through the film) were found at certain locations on the DLC specimen surface (Fig. 3(a)). The transfer film in Fig. 3(a) was discontinuous not only in the horizontal direction as shown in the photo but also in the vertical or sliding direction, which is not shown here (but is shown schematically in Fig. 4).

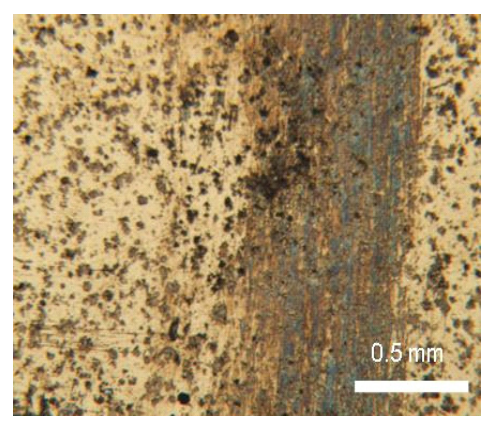

(a) $\mathrm{DLC}$ on $\mathrm{Al}_{2} \mathrm{O}_{3}$

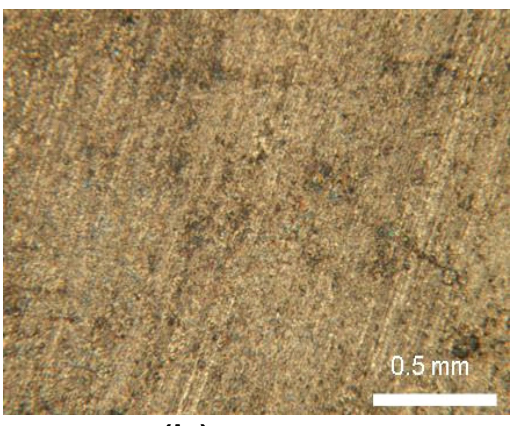

(b) $\mathrm{S} 45 \mathrm{C}$

Fig. 3 Tested DLC and non-DLC specimen surfaces

\section{Discussion}

Although we expected that exfoliation of DLC would not occur easily in the experiment due to a contact pressure not so high, exfoliation occurred early in the tests. This is probably due to the high, fluctuating friction of the DLC specimens, which exerted a high, fluctuating shear stress on the interface between DLC and the substrate.

The partially distributed transfer film shown in Fig. 3(a) could possibly affect the high, fluctuating friction and high frictional noise. The local friction coefficient was measured using the upper and lower specimens shown in Fig. 4 in order to distinguish the friction coefficient of transfer and non-transfer areas on the DLC surface. The lower specimen was cut down except for one part $5 \mathrm{~mm}$ in length, and this intact 5 - $\mathrm{mm}$ part slid on the tested upper DLC specimen, which had transfer films at certain locations on its surface. 


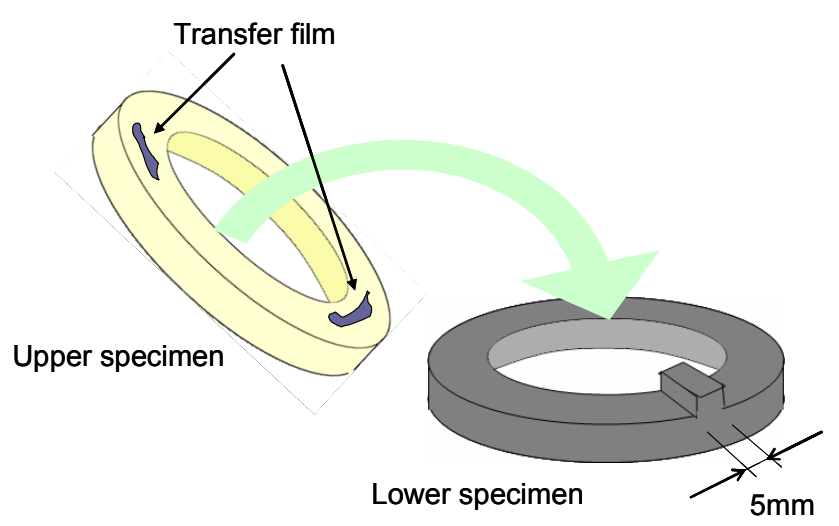

Fig. 4 Upper and lower specimens for measuring local friction coefficient

Figure 5 shows the friction coefficient distribution on the DLC surface. The friction coefficient on the transfer films showed a higher value than that on the non-transfer areas. This locally different friction coefficient distribution probably generated the high frictional noise.

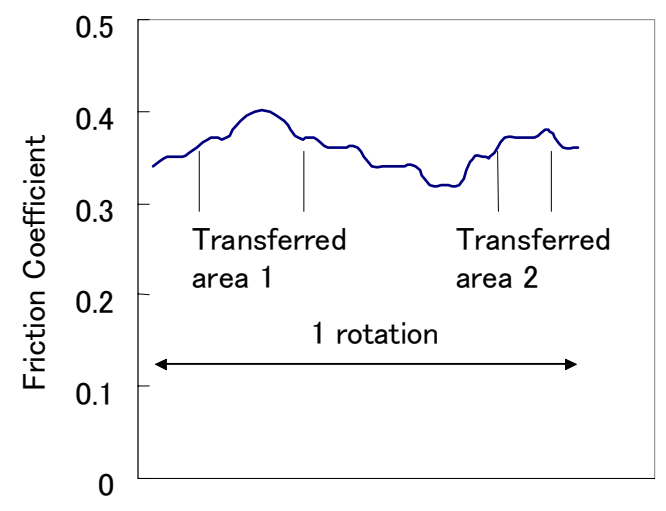

Fig. 5 Friction coefficient distribution on DLC surface

Graphite generally produces transfer film when exerting good tribological performance ${ }^{(9),(10)}$, such as its low friction coefficient when sliding on transfer film. The test results, however, showed the opposite tendency. Figure 6 shows the surface profiles of the DLC and non-DLC upper specimens before the tests. The DLC surface had some peaks of roughness made in the film deposition process probably due to defects or particles on the DLC surface being processed. Such peaks were not found on the non-DLC surface, though general amplitudes of roughness were almost same between both surfaces. This finding suggests the possibility of these peaks causing the high friction by ploughing and with the films being selectively transferred around the peaks.

A lightly lapped DLC specimen on which peaks of roughness were not found was tested. Fig. 2(a) also shows the result. Although the friction appeared to fluctuate less than that of the non-lapped DLC specimens, the friction coefficient increased in several tens of minutes after the test began along with the generation of high frictional noise, which led to the test being discontinued. Transfer films similar in appearance to those on the non-lapped DLC specimens were found on the tested surface. These results suggest that the peaks of roughness found on the non-lapped DLC surface did not directly cause the high friction (though could be considered one cause of fluctuating friction). 

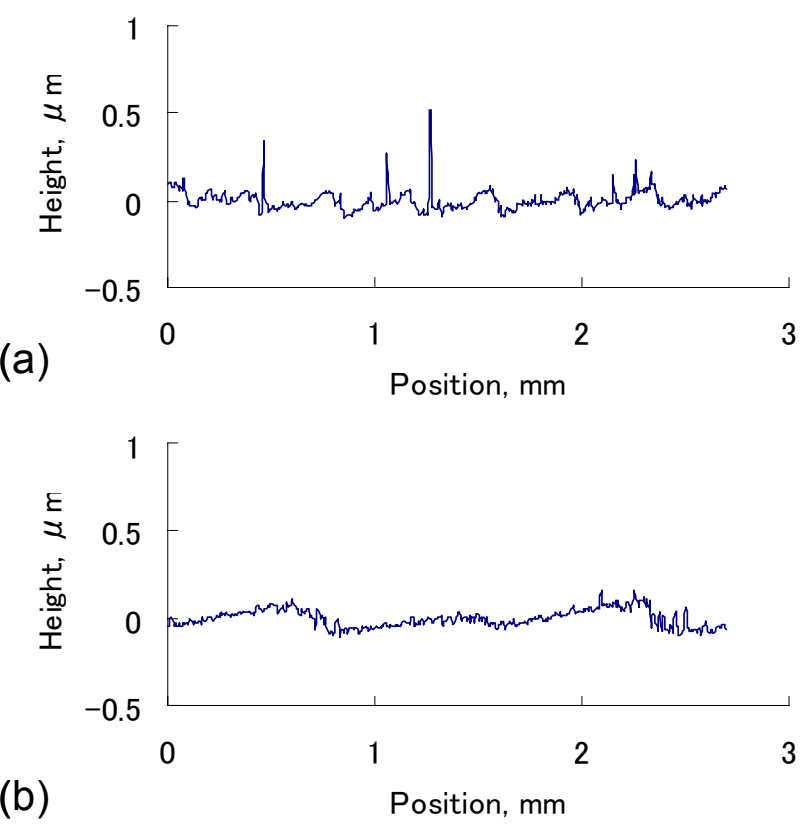

Fig. 6 Surface profiles of DLC and non-DLC upper specimens before the tests (a) DLC (b) Non-DLC $\left(\mathrm{Al}_{2} \mathrm{O}_{3}\right)$

The above results suggest that the transfer film itself on the DLC surface was the major cause of increased friction and high frictional noise. A similar case was reported in Ref. (11), where polyimide sliding on DLC showed high and unstable friction, and polyimide wear debris easily adhered to the DLC surface due to the high structural compatibility between DLC and polyimide, which had a plate-like shape and acted as an abrasive. In our experiment, transfer film did not seem abrasive. PTFE was also reported to form transfer film on DLC ${ }^{(12),(13)}$, where the film reduced friction in the same manner as typical PTFE friction. In our experiment, however, the transfer film increased the friction, which is behavior contrary to that of usual graphite friction.

Since the transfer film from sintered carbon to the non-DLC surface did not increase friction while that to the DLC surface did increase friction, and these films were considerably different in appearance, the film structures were possibly different. The films were subject to laser Raman spectrum analyses (employing a 532-nm wavelength YAG laser, $2 \mu \mathrm{m}$ in spot diameter) to investigate the difference in the order of crystallite orientation between the structures of those films.

Figure 7 shows the Raman spectrums of the transfer films. The spectrums of orthogonal directions on the films were analyzed, where one direction was approximately parallel to the sliding direction and the other perpendicular to it. The spectrums of transfer films on both the DLC and non-DLC surfaces were almost the same and no directional difference was observed. All spectrums for the transfer films had two peaks: one (designated the "G-band") was located around $1600 \mathrm{~cm}^{-1}$ in Raman shift that originated from a ordered graphite structure; the other (designated the "D-band") was located around 1300 1400 $\mathrm{cm}^{-1}$ and originated from a disordered, defective, or very fine graphite crystallite structure ${ }^{(14)}$. The results showed that both transfer films had nearly the same shapes of "G-band" and "D-band" peak combinations, that is, both were composed of nearly the same ordered and disordered mixed structure. 


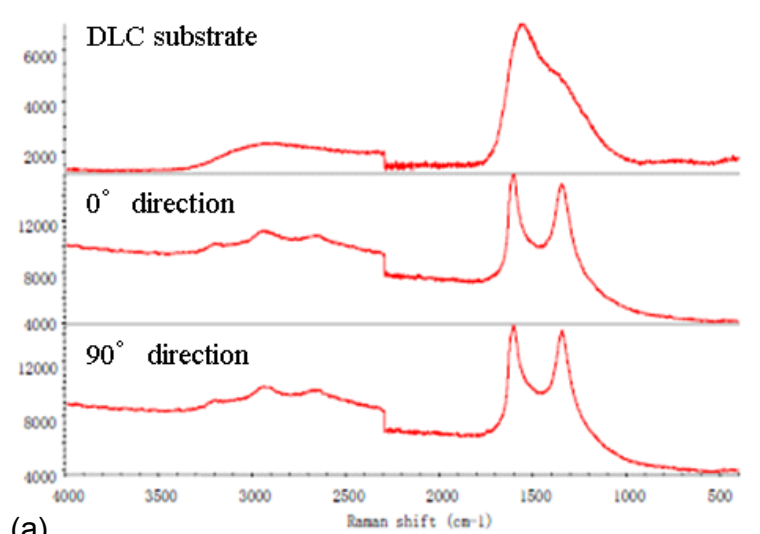

(a)

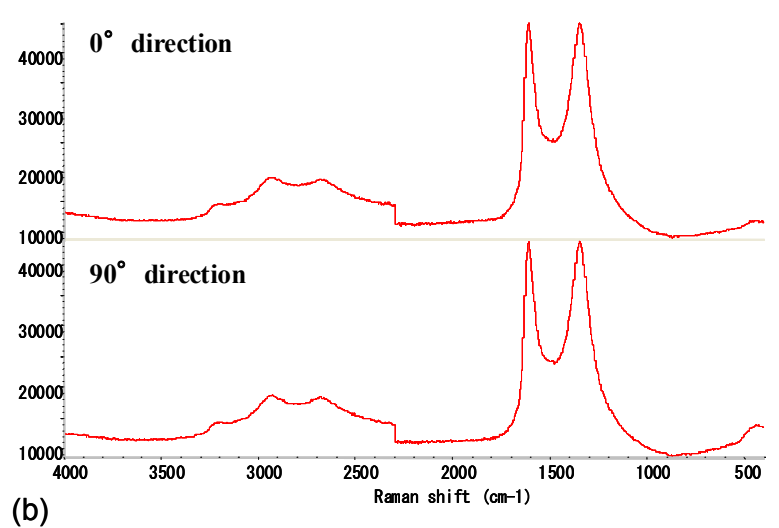

Fig. 7 Raman spectrums of transfer films: (a) film on DLC (b) film on non-DLC $\left(\mathrm{Al}_{2} \mathrm{O}_{3}\right)$ The $0^{\circ}$ and $90^{\circ}$ directions refer to the sliding direction and perpendicular direction thereto, respectively.

As described above, the difference in structural order between the transfer films on the DLC and non-DLC surfaces was not detected by Raman spectrum analyses. However, the spectrums provided information about the surface layers down to the depth mostly equivalent to laser wavelength, that is, the spectrums did not provided information about "just the surface" and there was a possibility that the components of the film material (e.g. percentage of resin content) were different. In particular, the phenolic resin impregnant in the sintered carbon was likely to adhere to the DLC surface, similar to polyimide in Ref. (11). However, this was not the main cause of high friction because the sintered-carbon impregnant with antimony also showed increased friction. Nevertheless, there is still a possibility of the transfer film playing some role in generating high friction.

Next, we focused on the sintered-carbon surface. Figure 8 shows photos of the tested sintered-carbon specimen surfaces having slid on the DLC and non-DLC surfaces. The specimen surface having slid on the non-DLC surface appears to have a strongly oriented surface layer in the sliding direction, while that on the DLC surface showed little traces of sliding and looked like the pre-tested specimen surface. The contact angles of pure water with respect to the tested sintered-carbon surfaces having slid on the DLC and non-DLC surfaces were 65 deg. and $77 \mathrm{deg}$., respectively. The sintered-carbon surface having slid on the DLC surface had a slightly higher hydrophilic property than that on the non-DLC surface, which probably means that the surface free energy of the sintered-carbon surface having slid on DLC was slightly larger than that on the sintered-carbon surface having slid on the non-DLC surface. 


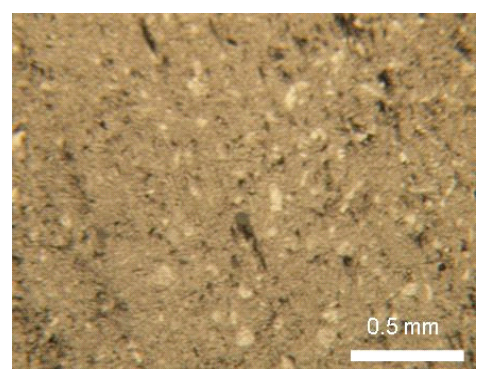

vs. DLC

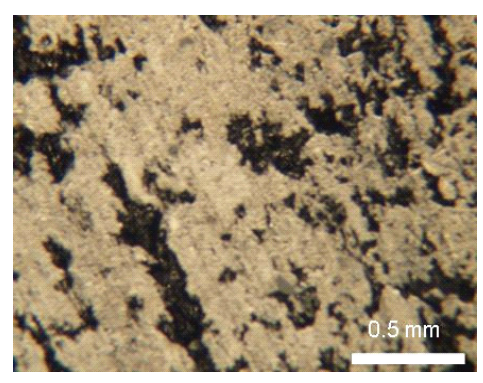

vs. Non-DLC

Fig. 8 Tested sintered-carbon specimen surfaces

These results suggest the following mechanism of generating large friction in the DLC and sintered-carbon sliding pair. Although sintered carbon did not easily form transfer film on DLC due to the anti-adhesion property of the DLC surface, it formed transfer films at some locally active sites of DLC due to defects or other causes. The orientation of the sintered-carbon surface during sliding did not progress much because most of the mated surface was the non-transferred DLC and the amorphous, anti-adhesion DLC surface did not strongly or forcibly orient the sintered-carbon surface in the sliding direction.

Since the transfer film showed a volume of disordered structure, as verified by Raman spectrum analyses, many edges or defects of graphite crystallites were exposed on the film surface. When the little-oriented sintered-carbon surface, on which many edges or defects of graphite crystallites, amorphous carbon, and impregnants were exposed, slid on the transfer film surface, these edges or defects on both surfaces interacted strongly and generated higher friction than that when sliding on the non-transferred DLC surface. In the highly oriented sintered-carbon surface, which generated friction upon sliding on the non-DLC surface, the surface layer was probably predominated by basal planes of graphite crystallites, and the surface free energy was lower than that of the low-oriented surface, as evidenced by the contact angles of water. The non-DLC and sintered-carbon sliding pair showed relatively low friction probably due to this highly oriented sintered-carbon surface.

\section{Conclusions}

Sintered carbon was found to generate high friction and high frictional noise upon sliding on DLC materials. This high friction induced the exfoliation of DLC from the substrate. Although the DLC and sintered-carbon sliding pair is a compatible carbon-carbon material combination, transfer films of sintered carbon to the DLC surface were only formed at certain locations on DLC, while most surface of the non-DLC materials was covered by transfer film for the non-DLC and sintered-carbon sliding pair. The high friction of the DLC resulted from the friction generated between the sintered carbon and transfer film, but not that generated between the sintered carbon and DLC itself. The sintered-carbon surface having slid on the DLC surface was little oriented while that on the non-DLC surface was strongly oriented to the sliding direction. Thus, the sintered graphite carbon sliding on the transfer film generated high friction when its surface was little oriented, which was the major cause of the high friction of DLC.

As a result, DLC and sintered-carbon combination was found not to be a preferable material combination for mechanical seals in dry gas sealing applications under such sliding conditions as this study. 


\section{References}

(1) Japanese Society of Tribologists ed., Minor Special Issue on DLC and Diamond Films, Journal of Japanese Society of Tribologists, Vol.47, No.11 (2002), pp.795-845.

(2) Otake, N. and Nakahigashi, T. ed., Handbook of Diamond-Like Carbon, (2006), N.T.S., pp.131-232.

(3) Kano, M., Application and Technical Issues of DLC Coating, Journal of Japanese Society of Tribologists, Vol.52, No.3 (2007), pp.186-191.

(4) Otake, N. and Nakahigashi, T. ed., Handbook of Diamond-Like Carbon, (2006), N.T.S., pp.68-69.

(5) Sheeja, D., Tay, B. K., Krishnan, S. M., and Nung, L. N., Tribological Characterization of Diamond-Like Carbon (DLC) Coatings Sliding Against DLC Coatings, Diamond and Related Materials, Vol. 12, No.8 (2003), pp. 1389-1395.

(6) Sheeja, D., Tay, B. K., and Nung, L. N., Tribological Characterization of Surface Modified UHMWPE Against DLC-Coated Co-Cr-Mo, Surface and Coatings Technology, Vol. 190, No.2-3 (2005), pp. 231-237.

(7) Kennedy, F. E., Lidhagen, D., Erdemir, A., Woodford, J. B., and Kato, T., Tribological behavior of hard carbon coatings on steel substrates, Wear, Vol. 255, No.7-12 (2003), pp. 854-858.

(8) Maeda, E., Miyazaki, K., Shibata, K., and Yoshida, H., Friction and Wear Between Carbon Materials at High Temperatures, Tanso, No.162 (1994), pp. 96-99.

(9) Zaidi, H., Robert, F., and Paulmier, D, Influence of adsorbed gases on the surface energy of graphite: consequences on the friction behaviour, Thin Solid Films, Vol.264, No.1 (1995), pp.46-51.

(10) Japanese Society of Tribologists ed., Tribology Handbook, (2001), Yokendo, pp.736-742.

(11) Samyn, P., Schoukens, G.., Quintelier, J., and De Baets, P., Friction, Wear and Material Transfer of Sintered Polyimides Sliding Against Various Steel and Diamond-Like Carbon Coated Surfaces, Tribology International, Vol.39, No.6 (2006), pp.575-589.

(12) Yang, E. L., Anttila, A., Hirvonen, J. P., and Toivanen, R. O., Friction and Wear of Polytetrafluoroethylene on Diamond-Like Carbon Film, Thin Solid Films, Vol.196, No.1 (1991), pp.L25-L29.

(13) Yang, E. L. and Hirvonen, J. P., Tribological Transfer of Polytetrafluoroethylene onto a Diamond-Like Carbon Film, Thin Solid Films, Vol.226, No.2 (1993), pp.224-229.

(14) Tuinstra. F., and Koenig, J. L., Raman Spectrum of Graphite, Journal of Chemical Physics, Vol.53, No.3 (1970), pp.1126-1130. 